\title{
The Neglected Role of Trichomonas tenax in Oral Diseases: A Systematic Review and Meta-analysis
}

Aida Vafae Eslahi ${ }^{a}$, Meysam Olfatifar ${ }^{\mathrm{b}}$, Amir Abdolic, Elham Houshmand ${ }^{\mathrm{d}}$, Morteza Ghanbari Johkool ${ }^{\mathrm{a}}$, Mahdieh Zarabadipour ${ }^{\mathrm{e}}$, Pegah Afsaneh Abadi ${ }^{\mathrm{a}}$, Azam Ghorbani ${ }^{\mathrm{a}}$, Monirsadat Mirzadeh ${ }^{\mathrm{f}}$, Milad Badri ${ }^{\mathrm{a}}$

${ }^{a}$ Metabolic Diseases Research Center, Research Institute for Prevention of Non-Communicable Diseases, Qazvin University of Medical Sciences, Qazvin, Iran

${ }^{\mathrm{b}}$ Gastroenterology and Liver Diseases Research Center, Research Institute for Gastroenterology and Liver Diseases, Shahid Beheshti University of Medical Sciences, Tehran, Iran

${ }^{\mathrm{c}}$ Department of Parasitology and Mycology, School of Medicine, Jahrom University of Medical Sciences, Jahrom, Iran

${ }^{\mathrm{d}}$ Department of Parasitology, Faculty of Veterinary Medicine, Rasht branch, Islamic Azad University, Gilan, Iran

${ }^{\mathrm{e}}$ Oral Medicine Department, School of Dentistry, Qazvin University of Medical Science, Qazvin, Iran

${ }^{\mathrm{f}}$ Community Medicine, Metabolic Diseases Research Center, Research Institute For Prevention Of NonCommunicable Diseases, Qazvin University Of Medical Sciences, Qazvin, Iran

corresponding author: Badri, M.; Metabolic Diseases Research Center, Research Institute for Prevention of Non-Communicable Diseases, Qazvin University of Medical Sciences, Qazvin, Iran;

email:Badri22.milad@gmail.com

\begin{abstract}
Purpose: Trichomonas tenax (T. tenax) is a commensal flagellated protozoan found in periodontal microenvironment of the oral cavity, with a possible role in periodontal diseases. The purpose of the present systematic review and meta-analysis was to determine the worldwide prevalence of $\mathrm{T}$. tenax infection and to show the neglected association of this parasitic infection with oral diseases. Methods: To find literatures published until August 2020, five English databases (PubMed, Scopus, Science Direct, Web of Science and Google Scholar) were explored. Finally, 65 papers were qualified to be included in the current study. Results: Our results revealed a global pooled prevalence of $17 \%$ (95\% CI 14-22\%) for T. tenax infection. The highest prevalence was estimated at 56\% (42-69\%) in Chile, while the lowest prevalence was related to Kenya with 3\% (1-6\%). The age-based analysis found that the infection was most common in 46-55 mean age group with $15 \%(0-100 \%)$. The overall prevalence regarding culture method, molecular method and direct method was $21 \%(12-32 \%), 19 \%(8-35 \%)$ and 17\% (12-23\%), respectively. Moreover, the subgroup analysis showed the pooled prevalence in patient with candidiasis [22\% (3-52\%)], gingivitis $[21 \%(9-36 \%)]$ and periodontitis [27\% (10-48\%)]. Conclusion: Our study specified a connection between T. tenax protozoa and periodontitis disease. However, more epidemiological studies as well as clinical trials are needed to precisely identify this relation. (C) 2021, Witold Stefański Institute of Parasitology, Polish Academy of Sciences.
\end{abstract}

Author keywords

Oral health; Prevalence; Trichomonas tenax; Worldwide 